\title{
Lewis Acid-Water/Alcohol Complexes as Hydrogen Atom Donors in Radical Reactions
}

\author{
Guillaume Povie ${ }^{\S}$ and Philippe Renaud ${ }^{*}$ \\ §SCS-Metrohm Foundation Award for best oral presentation
}

\begin{abstract}
Water or low molecular weight alcohols are, due to their availability, low price and low toxicity ideal reagents for organic synthesis. Recently, it was reported that, despite the very strong BDE of the O-H bond, they can be used as hydrogen atom donors in place of expensive and/or toxic group 14 metal hydrides when boron and titanium(III) Lewis acids are present. This finding represents a considerable innovation and uncovers a new perspective on the paradigm of hydrogen atom transfers to radicals. We discuss here the influence of complex formation and other association processes on the efficacy of the hydrogen transfer step. A delicate balance between activation by complex formation and deactivation by further hydrogen bonding is operative.
\end{abstract}

Keywords: Boron · Hydrogen atom donor - Hydrogen transfer - Lewis acids - Radical reactions . Solvent effect $\cdot$ Titanium

\section{Introduction}

The $\mathrm{O}-\mathrm{H}$ bond in water and alcohols is one of the strongest single bonds in organic molecules $\left(\mathrm{BDE}=110-118 \mathrm{kcal} \mathrm{mol}^{-1}\right){ }^{[1]}$ Such high bond energies normally preclude hydrogen atom transfer to organic free radicals, and the hydroxyl group of alcohols is generally considered to be inert in radical mediated transformations. In contrast to this commonly accepted behaviour, several groups recently reported reductive radical transformations in which the transferred hydrogen atom originated from the hydroxyl group of water or alcohols.

Using trivalent titanocene complexes to generate $\beta$-titanoxy alkyl radicals from epoxides, ${ }^{22}$ Cuerva, Oltra and coworkers observed the formation of the reduced compound when the reaction was performed in wet tetrahydrofuran (Scheme 1). [3] Since hydrogen atom transfer from water was deemed unfeasible, hydrolysis

${ }^{*}$ Correspondence: Prof. Dr. P. Renaud Universität Bern

Departement für Chemie und Biochemie

Freiestrasse 3

$\mathrm{CH}-3012$ Bern

Tel.: +41316314359

E-mail: philippe.renaud@ioc.unibe.ch of an organotitanocene intermediate was first proposed. Nevertheless, extensive deuteration and competition experiments discarded this hypothesis, and water was eventually recognised as the source of hydrogen atoms in this reaction. ${ }^{[4]}$ This dramatic weakening of the water $\mathrm{O}-\mathrm{H}$ bond was reasonably attributed to the formation of a Lewis acid-base complex with the transition metal (Scheme 1, complex
A), ${ }^{[5]}$ for which DFT calculations showed a diminution of the $\mathrm{O}-\mathrm{H}$ bond dissociation energy (BDE) by nearly $60 \mathrm{kcal} \mathrm{mol}^{-1} \cdot{ }^{[4,6]}$

In parallel, Wood and coworkers observed a similar behaviour of water in trimethylborane mediated BartonMcCombie deoxygenations (Scheme 2). ${ }^{[7]}$ Supported by DFT calculations, they postulated that the formation of a boronate complex $\mathbf{B}$ between the trialkylborane and

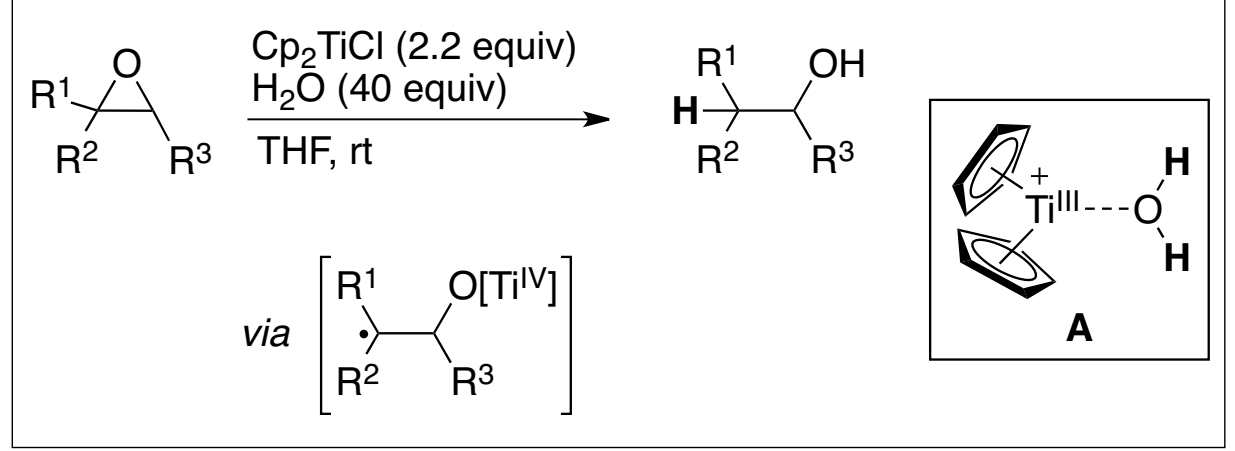

Scheme 1. Radical reduction of epoxide using $\mathrm{Cp}_{2} \mathrm{TiCl}-\mathrm{H}_{2} \mathrm{O}$.

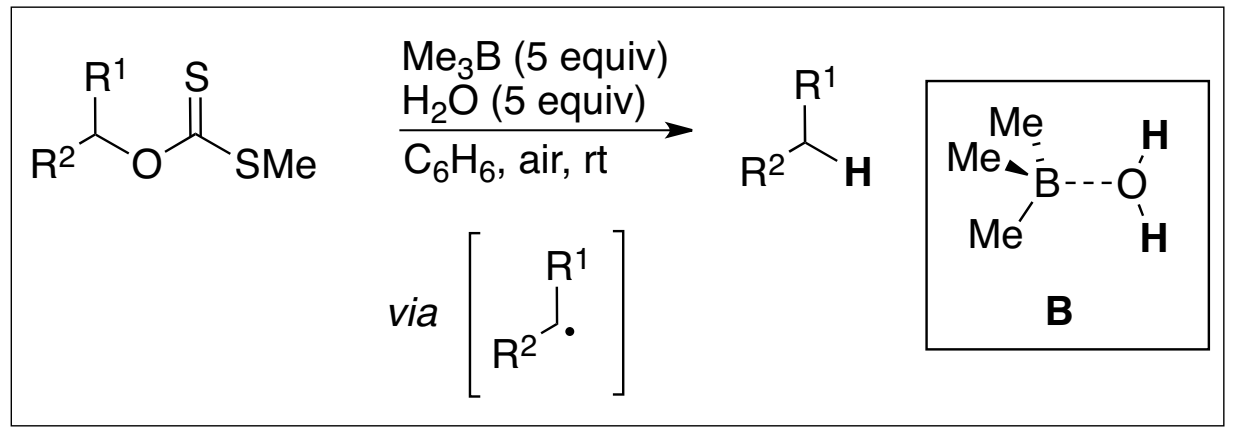

Scheme 2. Radical reduction of xanthates using $\mathrm{Me}_{3} \mathrm{~B}-\mathrm{H}_{2} \mathrm{O}$.[7] 


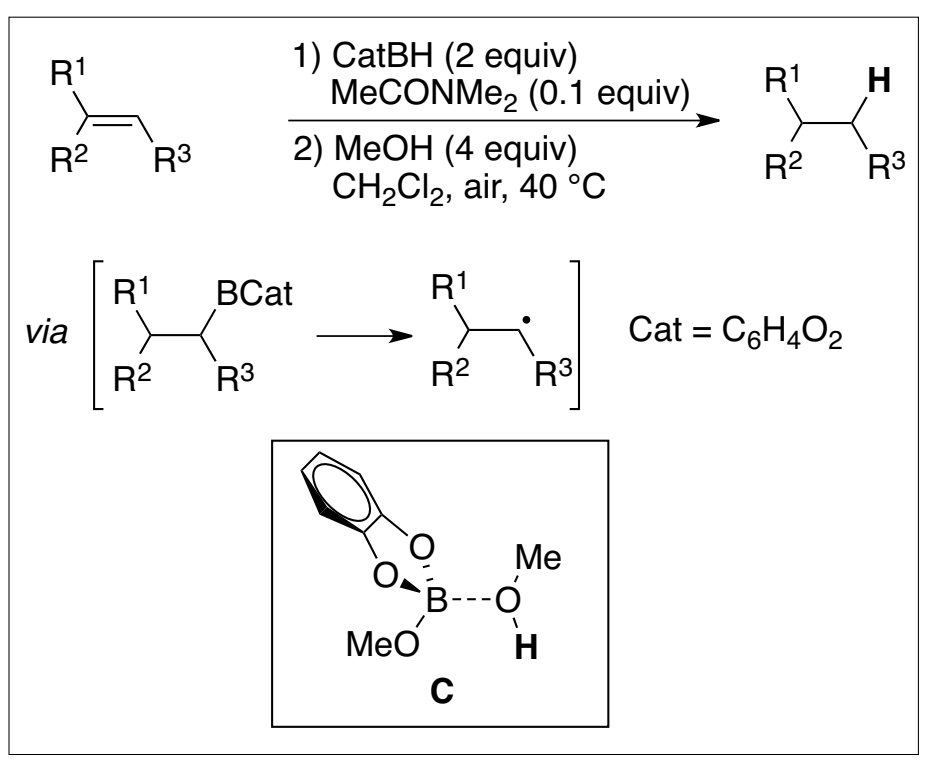

Scheme 3. Reduction of alkenes via hydroboration/radical protodeboration. ${ }^{[9 a]}$ water would drastically decrease the $\mathrm{O}-\mathrm{H}$ BDE. This process could then be extended to various reductive transformations, using different combinations of primary trialkylboranes and alcohols as hydrogen-deuterium sources. ${ }^{[8]}$

During the same years, our group disclosed a method for the radical protonolysis of $B$-alkylcatecholboranes using methanol as the hydrogen atom source (Scheme 3). ${ }^{[9]}$ The activation of the $\mathrm{O}-\mathrm{H}$ bond in methanol by complexation with the Lewisacidic $B$-methoxycatecholborane was also proposed, however no direct evidence for the formation of such species could be obtained (Scheme 3, complex $\mathbf{C}$ ).

\section{Complex Formation}

Water or low molecular weight alcohols are certainly the cheapest molecules, and their use as radical reducing agents in place of expensive and/or toxic group 14 metal hydrides would represent a considerable innovation. In a broader view, this unprecedented mode of activation might have important consequences on the mechanism of biological enzymatic transformations, where transition metal-aqua complexes are involved. The answer to whether or not this behaviour is operative in other systems requires a fundamental understanding of the parameters governing it, and to start addressing this question, both thermodynamic information on the association process and kinetic data on the rate of the hydrogen atom transfer are essential.

Studying the Lewis acid-base interactions between titanocene and various ligands by UV spectroscopy, Cuerva and coworkers found the formation of the titanium-aqua complex $\mathbf{A}$ to be already quantitative at rather low concentrations of water (ca. 0.1 M in THF). ${ }^{[10]}$ In parallel, Newcomb and Jin measured the rate $k_{\mathrm{H}}$ at which complex A transfers a hydrogen atom to a secondary alkyl radical to be about $10^{5} \mathrm{M}^{-1} \mathrm{~s}^{-1}$ at $293 \mathrm{~K}$, a value close to the one measured for tris-trimethylsilylsilane, a commonly used hydrogen atom donor in radical reactions. [11]

On the other hand, despite the widespread use of boron derivatives as Lewis acids, little is known about their association with alcohols. ${ }^{[12]}$ Hence, to gain further information on these putative reactive mation of organoboranes-methanol complexes using ${ }^{11} \mathrm{~B}$ NMR spectroscopy. In the case of $B$-methoxycatecholborane-methanol mixtures, no tetracoordinated species could be detected under the reaction conditions, strongly discarding the implication of complex $\mathbf{C}$ in the reduction process. ${ }^{[13]}$ Instead, we observed a rapid transesterification reaction with methanol that generated trimethylborate and catechol (Scheme 4). The latter was then confirmed to be the genuine hydrogen atom donor, leading to the development of a mild procedure for the formal protonolysis of organoboranes. ${ }^{[14]}$

Interested by the comparative behaviour of trialkylboranes, we next focused intermediates, we started to study the for- with methanol when employing similar concentrations as used in kinetic studies of the hydrogen atom transfer to a secondary alkyl radical.[15a] This result advocates for a much more important activation of the $\mathrm{O}-\mathrm{H}$ bond of methanol by a trialkylborane than previously envisaged. Using the actual concentration of complex, the rate constant for the hydrogen atom transfer is found to be about $10^{6} \mathrm{M}^{-1} \mathrm{~s}^{-1}$, a value similar to the one reported for tri- $n$-butyltin hydride.

\section{Hydrogen Bonding Environment}

At first sight, these results suggest that in pure methanol solution at room temperature, an excellent hydrogen atom donating ability should be observed since Et $\mathrm{B}$ is expected to be quantitatively complexed by methanol. This is however not the case; $\mathrm{Et}_{3} \mathrm{~B}$ mediated iodine atom transfer reactions are known to take place efficiently in water and alcohols without formation of reduced products. ${ }^{[17]}$ This counterintuitive result is best explained by hydrogen bonding. The effect of hydrogen bonding on hydrogen atom transfer reactions has been thoroughly studied in the case of phenols, leading to a quantitative description of kinetic solvent effects. ${ }^{[18]}$ In the presence of hydrogen bond acceptors, only the phenols free of hydrogen bonding can transfer their hydrogen atoms. ${ }^{[19]}$ As all hydroxylic compounds methanol is prone to form hydrogen bonds, being principally in the form of poly/oligomeric clusters in solution. ${ }^{[20]}$ Upon complexation with a Lewis acid, a water or alcohol molecule should not lose its hydrogen bond donating ability. Therefore, the environment of such complexes has a dramatic effect on their aptitude to transfer hydrogen atoms. Zipse's calculations on the trimethylborane-water complex $\mathbf{B}$ already suggested the detrimental effect of the coordination of a second water molecule. ${ }^{[21]}$ Similarly, hydrogen bond formation with THF has recently been proposed to dramatically decrease the reactivity of the titanocene-water complex A. ${ }^{6]}$ Thus,

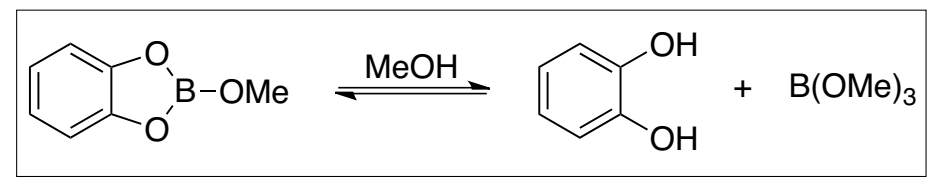

Scheme 4.

Transesterification of $B$-methoxycatecholborane with methanol.

our attention on the coordination of methanol by triethylborane $\left(\mathrm{Et}_{3} \mathrm{~B}\right)$, a system for which the rate of hydrogen atom transfer had previously been studied by Newcomb and Jin. ${ }^{[15]}{ }^{11} \mathrm{~B}$ NMR spectroscopy revealed that $\mathrm{Et}_{3} \mathrm{~B}$ and methanol associate, but only to a minor extent under the reported reaction conditions (Scheme 5). ${ }^{[16]}$ For instance, only 1-2\% of $\mathrm{Et}_{3} \mathrm{~B}$ was complexed

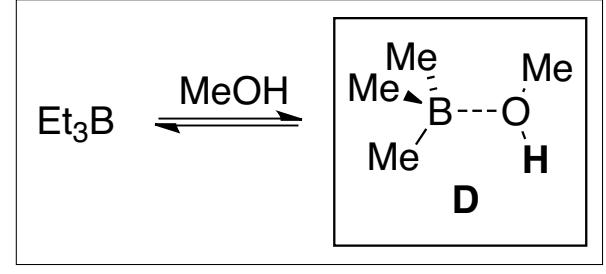

Scheme 5 . $\mathrm{Et}_{3} \mathrm{~B}-\mathrm{MeOH}$ equilibrium association. $K_{299 \mathrm{~K}}=0.08 \mathrm{M}^{-1}$. 


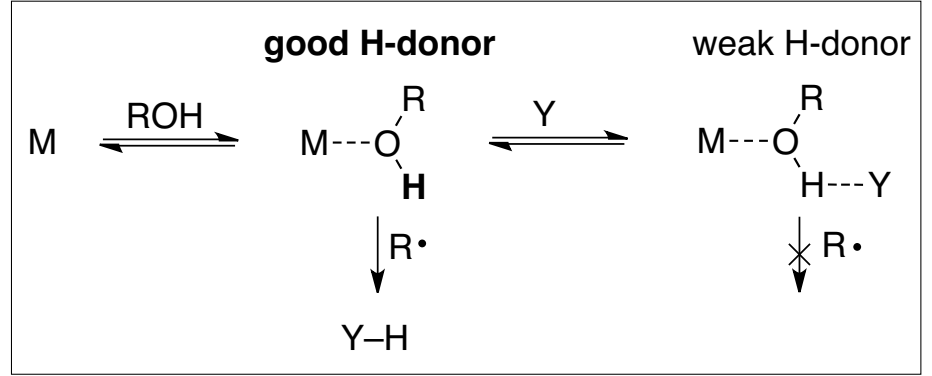

Scheme 6. Activation of water and alcohol toward hydrogen atom transfer by Lewis acids $\left(M=R_{3} B\right.$, $\mathrm{Cp}_{2} \mathrm{TiCl}$ ) and deactivation of the corresponding complex by further hydrogen bonding $(\mathrm{Y}=\mathrm{ROH}$, THF...).

the rate constants determined for both complex $\mathbf{A}$ and $\mathbf{D}$ are probably underestimated since only the fraction of complex not involved in further hydrogen-bonding will react (Scheme 6). The reactivity of the free complexes for hydrogen atom transfer reactions is thus probably much higher that initially thought, in accordance with the corresponding low calculated $\mathrm{O}-\mathrm{H}$ bond dissociation energies. ${ }^{[6,7]}$

\section{Conclusions}

In solutions, further hydrogen-bonding of metal-aqua complexes may have masked the activation of water toward hydrogen atom transfer, which could then be more general than the two cases reported until now. The question of which type of Lewis acid could induce a large diminution of the $\mathrm{O}-\mathrm{H}$ bond dissociation energy upon complexation with an alcohol or water remains opened. Seen as a proton coupled electron transfer, the hydrogen atom transfer requires an electron rich orbital available to furnish the electron. Titanium(III) is a well-known one-electron reducing agent whereas hyperconjugation of the electron rich $\mathrm{C}-\mathrm{B}$ sigma bond with the $\mathrm{O}-\mathrm{H}$ bond of water may fulfil this role in the case of trialkylborane complexes $\mathbf{B}$ and D. ${ }^{[21]}$ Albeit counter-intuitive, the common characteristic would thus be electron-rich
Lewis acids. The idea of associating a good proton donor with a good one-electron donor to generate an efficient hydrogen atom donor appears then fairly reasonable and opened the door to the development of new reagents.

Received: March 5, 2013

[1] Y. R. Luo, 'Handbook of bond dissociation energies in organic compounds', CRC Press, Boca Raton, FL, 2003.

[2] T. V. RajanBabu, W. A. Nugent, J. Am. Chem. Soc. 1994, 116, 986.

[3] A. F. Barrero, J. E. Oltra, J. M. Cuerva, A. Rosales, J. Org. Chem. 2002, 67, 2566.

[4] J. M. Cuerva, A. G. Campaña, J. Justicia, A. Rosales, J. L. Oller-López, R. Robles, D. J. Cárdenas, E. Buñuel, J. E. Oltra, Angew. Chem. Int. Ed. 2006, 45, 5522 .

[5] Although the first proposed structure consisted of a tetracoordinated titanium species, recent spectroscopic and computational studies supported a cationic intermediate where the chloride anion is dissociated, see ref. [6].

[6] A. Gansäuer, M. Behlendorf, A. Cangönül, C. Kube, J. M. Cuerva, J. Friedrich, M. van Gastel, Angew. Chem. Int. Ed. 2012, 51, 3266.

[7] D. A. Spiegel, K. B. Wiberg, L. N. Schacherer, M. R. Medeiros, J. L. Wood, J. Am. Chem. Soc. 2005, 127, 12513.

[8] a) M. R. Medeiros, L. N. Schacherer, D. A. Spiegel, J. L. Wood, Org. Lett. 2007, 9, 4427; b) F. Allais, J. Boivin, V. T. Nguyen, Beilstein J. Org. Chem. 2007, 3, No. 46; c) J. Boivin, V. T. Nguyen, Beilstein J. Org. Chem. 2007, 3, No. 45; d) J. Boivin, V. T. Nguyen, Beilstein J. Org. Chem. 2007, 3, No. 47; e) J. A. Davy, J. W. Mason, B. Moreau, J. E. Wulff, J. Org. Chem. 2012, 77, 6332 .
[9] a) D. Pozzi, E. M. Scanlan, P. Renaud, J. Am. Chem. Soc. 2005, 127, 14204; b) D. Pozzi, P. Renaud, Chimia 2007, 61, 151.

[10] M. Paradas, A.G. Campaña, T. Jiménez, R. Robles, J. E. Oltra, E. Buñuel, J. Justicia, D. J. Cárdenas, J. M. Cuerva, J. Am. Chem. Soc. 2010, 132, 12748.

[11] J. Jin, M. Newcomb, J. Org. Chem. 2008, 73, 7901.

[12] a) P. J. Domaille, J. D. Druliner, L. W. Gosser, J. M. Read, E. R. Schmelzer, W. R. Stevens, J. Org. Chem. 1985, 50, 189; b) D. Daniela, M. Daniela, B. Tiziana, D.A. Giuseppe, M. Pierluigi, S. Angelo, Eur. J. Inorg. Chem. 2008, 2008, 1645.

[13] G. Povie, G. Villa, L. Ford, D. Pozzi, C. H. Schiesser, P. Renaud, Chem. Commun. 2010, $46,803$.

[14] G. Villa, G. Povie, P. Renaud, J. Am. Chem. Soc. 2011, 133, 5913.

[15] a) J. Jin, M. Newcomb, J. Org. Chem. 2007, 72, 5098; b) J. Jin, M. Newcomb, J. Org. Chem. 2008, 73, 4740

[16] G. Povie, M. Marzorati, P. Bigler, P. Renaud, J. Org. Chem. 2013, 78, 1553.

[17] a) H. Yorimitsu, T. Nakamura, H. Shinokubo, K. Oshima, K. Omoto, H. Fujimoto, J. Am. Chem. Soc. 2000, 122, 11041; b) H. Yorimitsu, H. Shinokubo, S. Matsubara, K. Oshima, K. Omoto, H. Fujimoto, J. Org. Chem. 2001, 66, 7776; c) H. Yorimitsu, H. Shinokubo, K. Oshima, Synlett 2002, 5, 674; d) P. Panchaud, P. Renaud, J. Org. Chem. 2004, 69, 3205.

[18] a) L. R. C. Barclay, C. E. Edwards, M. R. Vinqvist, J. Am. Chem. Soc. 1999, 121, 6226; b) G. Litwinienko, K. U. Ingold, J. Org. Chem. 2004, 69, 5888; c) M. F. Nielsen, K. U. Ingold, J. Am. Chem. Soc. 2006, 128, 1172; d) G. Litwinienko, K. U. Ingold, Acc. Chem. Res. 2007, 40, 222.

[19] D. W. Snelgrove, J. Lusztyk, J. T. Banks, P. Mulder, K. U. Ingold, J. Am. Chem. Soc. 2001, $123,469$.

[20] a) M. Saunders, J. Hyne, J. Chem. Phys 1958, 29, 1319; b) J. C. Davis, K. S. Pitzer, C. N. R. Rao, J. Phys. Chem. 1960, 64, 1744; c) A. K. Chandra, A. B. Sannigrahi, J. Phys. Chem. 1965, 69, 2494; d) J. L. Kirsch, D. R. Coffin, J. Phys. Chem. 1976, 80, 2448; e) M. A. Wendt, J. Meiler, F. Weinhold, T. C. Farrar, Mol. Phys. 1998, 93, 145; f) H. Shekaari, H. Modarress, N. Hadipour, J. Phys. Chem. A 2003, 107, 1891.

[21] W. Tantawya, H. Zipse, Eur. J. Org. Chem. 2007, 5817. 